\title{
Mathematical and Trade based optimization : "synthetic" method and three industrial cases
}

\author{
Yvon Gardan \\ CReSTIC \\ URCA (Reims university) \\ Reims, France \\ gardan@infonie.fr
}

\author{
Nicolas Gardan \\ DINCCS \\ MICADO \\ Charleville-Mézières, France
}

Received: January 17, 2021. Revised: March 5, 2021. Accepted: March 17, 2021. Published: March 19, 2021.

\begin{abstract}
The linear process of design-engineeringmanufacturing is nowadays obsolete. Even DFX (Design For X) methodologies are often replaced by Simulation for XFD (X being also Design) approaches. Optimization is also often applied before design. For instance, engineering design optimization of mechanical structures is nowadays essential in the mechanical industry (automotive, aeronautics ...). But optimizing mechanical structures cannot be efficient without taking in account other phases of the design-manufacturing process. In some cases, optimization is only based on trade kwnowledge.
\end{abstract}

Engineers must then design parts or assemblies that are a better compromise, between mechanical and functional performance, weight, manufacturing costs etc.

In this paper, after giving an overview on the different disciplines in design optimization of mechanical structures, we propose some trends to take into account the trade knowledge in the optimization/design phases.

We propose an integrated approach to optimization in a functional design process with a methodological point of view. We present three different industrial cases of optimization on three examples : one in mechanical structures optimization, on based only on trade knowledge and the third mixing calculus and knowledge.

Keywords-engineering, design, optimization

\section{INTRODUCTION}

The optimization of various processes involved in the product life cycle management becomes more and more crucial. [1] and [2] show that the first phases of design take over $5 \%$ of the design process, but involve more than $75 \%$ of the total cost of the product. We can deduce that optimization in the design phase are essential in the product life cycle.

The research of the best compromise between economic, technological and mechanical parameters has always been the objective number one of the mechanical engineer. It has been for a long time based on a try / error approach. The development of techniques for solving mathematical problems and their implementation in algorithms change the context. It leads to new approaches and methodologies in the very first design phases.

As we have illustrated in [3], the classical ("manual") stages of design (achievement of one or several CAD models from the experience of engineer which respect to functional specifications, and the general environment of the structure, followed by the implementation of various calculation models (static, vibration, dynamic etc.) that assess the various design criteria of the structure and several iterations to change the design in order to meet the specifications and optimize the structure) is more and more often replaced by an approach integrating an optimization phase, especially the optimization of mechanical structures.

It is supposed to take into account design requirements and constraints (manufacturing constraints, constraints related to business processes, thermo mechanical performance, weight requirements and cost, etc.) in agreement with all project stakeholders. The engineers can then automatically reproduce through modeling and numerical optimization software, the work that a designer achieved manually, by adding many advantages :

- scanning a wider design space,

- possibility to implement Design of Experiments (DOE) and thus create approximation functions if needed,

- opportunity to reach an optimum by using more efficient algorithms.

For instance, these «automatic» methods can find optimal solutions for the optimization of 
mechanical structures even if [4] think that, « in real life, identification of the optimum design of an industrial problem is often not possible because of the size of the problems and lack of knowledge». That's why in many cases just a part of the optimization problem is processed, for example, the design optimization of mechanical structures.

In the "real life" it is very dangerous to leaving aside the influence of optimization on the manufacturing process.

Obviously, the results of a mechanical structure can lead to a more costly product due to a complex manufacturing process, or even, the impossibility to manufacture.

But optimization cannot only be considered for mechanical structure. We illustrate three cases of trade optimization, one on process optimization, one on mechanical structures and the last on a specific manufacturing process, additive manufacturing.

After a brief presentation of methods and techniques used for solving optimization problems, we take the example of a heavy hook to show the mass gains. We do not look at the influence on manufacturing process in this kind of optimization as we have shown it in [3].

We then give just some ideas about the optimization of a process in the case of the forging process.

And we explain the importance of taking into account the entire manufacturing process with an example about additive manufacturing.

\section{OPtimization Problems}

In this section, we give an overview of two ways to optimize a product or a process. Before describing some approaches, we introduce a "global" vision of the design-manufacturing that we call "synthetic", based on the fact that local optimization are not always improving global performances.

The main one in design is based on the mechanical structures optimization and for example deterministic and probabilistic methods or evolutionary algorithms. After presenting the main methods, we do not detail the knowledge methods that we illustrate in a next section on an example.

\section{A. A "synthetic" point of view}

Our research intends to define a global model which places the process module in the center of the DMU. Process module manages proceeds which modify the product. Our methodology is working on a hierarchical framework presented [5]. This framework allows a top-down approach by defining functions in a high abstraction level and refining them in a low abstraction level.

The 4 'P's are defined according to
- Project module represents all the entities relating to the organization, the resources (human and equipment). Project module is defined in the Application Environment.

- Product module represents all information which characterizes product contents in a systematic way. A product has different representations according to the predefined abstraction levels.

- Proceed represents abstracted definitions related to a sequence of physical or virtual steps which lead to the modifications of the product.

- Process represents a succession of tasks whose implementation contributes to the modification of the product.

\section{B. Optimization problems categories}

Different categories of optimization problems depending on the objective function (for example, minimize the mass) and the constraints (for example, to support forces) can be defined :

- Linear programming problems which are optimization problems with only linear objective function and constraints. This is easy to solve according to the linearity of the functions used.

- Quadratic programming problems are optimization problems that have an objective with quadratic terms. The constraints in this case are linear functions made by equalities or inequalities.

- Non-linear optimization problems are optimization problems with objective functions and/or constraints having all some non-linearity.

- Stochastic optimization problems are optimization problem using random variables.

We can also classify the optimization problems depending on the nature of the variables : discrete optimization method, continuous optimization methods and mixed methods (discrete and continuous variables).

We present in the following paragraphs the most commonly used methods for several optimization problems. Fundamental and mathematics are not discussed here, details are available in the specialized literature $[6,7,8]$.

Methods for solving optimization problems can be divided into two major groups: deterministic and stochastic methods. Deterministic methods are more efficient when the evaluation of the objective function is very fast or when the function is known. The most complex cases such as problems with many local optima (multi-modal problem), or the case of non-differentiable functions are often managed by stochastic methods.

The enumerative methods are used to find all solutions in a given domain, mainly for discrete variables. In some cases, methods are used to divide a continuous domain in order to apply enumerative methods. These methods have the advantage 
of reaching the global optimum only by calculating the objective function. They need a significant number of calculations of the objective function so they are often used to refine the solutions of other optimization methods.

The analytical methods are are called "sensitivity analysis" and need continuous and differentiable functions. Their advantage is to reach the global optimum for convex problems. The results are highly dependent on the initial choice (initial design). In non convex problems, analytical methods find most of the times a local optimum and other methods are used to approach the global optimum.

The probabilistic methods are used to solve optimization problems with several local minima. Because of using random processes, several executions of the same program can give different solutions. These methods are numerous with several variants, we present here three of the most used.

The evolutionary algorithms are based on evolution. The principle of evolutionary algorithms is based on three operators: selection, crossover and mutation to find a population of individuals with the best values for the objective function..

Simulated Annealing method is based on an analogy of the annealing procedure in metallurgy (slow cooling of the metal to obtain a metal without default). In optimization, the annealing procedure defines the objective function as an energy which can reach a minimum (cooling process).

For [9], the neural networks are like a computer simulation model, having the capacity for adaptation, learning and generalization of information. Neural networks in optimization serve to interpolate between input data for learning and then generalize the knowledge. In more practical terms neural networks are non-linear statistical data modeling or decision making tools.

\section{Knowledge based optimization}

We also consider that to implement adapted CAE systems, we must take into account trade knowledge. It is by an integration of trade knowledge in the CAD system or in the specific developed software modules that we achieve our goal to improve the performance of the user.

Many work have been done on knowledge management. We emphasize on two main points :

- Ontologies : we think that it is very powerful to base the work on ontologies. This means that during the analysis phase of a new industrial problem, we use our internal tools (developed in EEP4LM project) to define the domain ontology. This ensure that the things are well defined between the end-user and us. We don't develop this point in this paper.

- We also introduce the notion of Graphonumerical Numerical Parameter (GNP). The computer representation is "'action_object(constraint/parameter)', A user can define a
GNP like "create_hole(-through)(diameter)', which is the translation of "create a through hole with diameter"' (a specific syntax has been developed for an intuitive use [10,11]). This GNP is linked to a scenario which defines this GNP. When a GNP is applied, the system constructs an implicit parameter which is the link between GNP and trade rules or constraints. For the precedent GNP, an implicit parameter called "diameter_- hole", is created. A link is done with the SBC if a rule or a constraint has a similar parameter. The following section presents how a GNP is decomposed according to the hierarchical architecture. A GNP has a different representation in each level of the hierarchical architecture of the KBS.

When a new application is taken into account, we use P4LM approach and specific tools.

First, we study the different processes and define the ontology. Then we look at commercial tools used (or that could be used) and specific developments to do, according to our methodology and our tools.

Even if we study the overall numerical chain, to be sure not to attain a local optimum which does not contribute to a global optimum, we are mainly interested in well closed steps of the design process in which the trade knowledge modeling can lead to important gains in quality, cost or delay.

This kind of application, with a development effort between a few days and weeks, depending on the complexity of the product and data, produces significant benefits, including: quote achieving very quickly (virtually of instantaneously), reliability of choices (quote is validated by the entire chain, including simulation, and not just vaguely evaluated), if the contract is obtained, the design is "almost" already complete. As a consequence, the formalization process (P4LM, ontology) is very interesting for the company, even if the software itself is not implemented. Many examples show that the design / manufacturing process, assumed perfectly controlled by the firm, is only very imperfectly known and often has different implementations based on operators or shadow areas. This preliminary study may make improvements, sometimes even without software development. It is important to remain attentive to the consistency of approach compared to the global numerical chain and developments to undergo software (changes in materials, new practices, standards...).

In order to work with small and medium sized firms, we have developed a collaborative tool (Adhoc Collaboration). Its description is not a purpose of this paper, but it is interesting to know that this collaborative tool (asynchronous and synchronous ...) has been immediately adopted without any problem even by very small firms.

For example, CAD4SIM (CAD FOR SIMulation) [12] defines rules in order to help industrial firms, to better design in order to better simulate products. So, we define rules, to be used during the design process (automatically or manually), that 
ensure correct simulation. Let's take a first simple industrial case.

Figure 1 illustrates a zone which does not respect some of our rules (a patch must have 4 sides, two patches must not overlap). The finite elements model presents some bad elements and some equivalence problems that have to be solved by the simulation specialist. A consequence can be that new bad elements can be created.
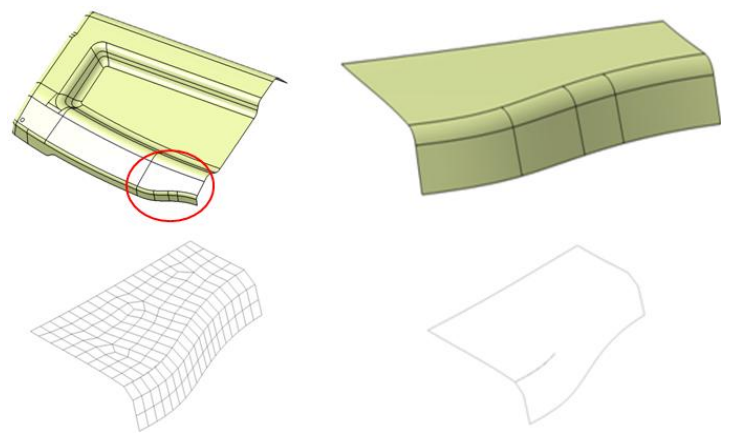

- $\quad$ Figure 1 : the initial design

Figure 2 shows modification using CAD4SIM rules. The FEM model is clearly of better quality.
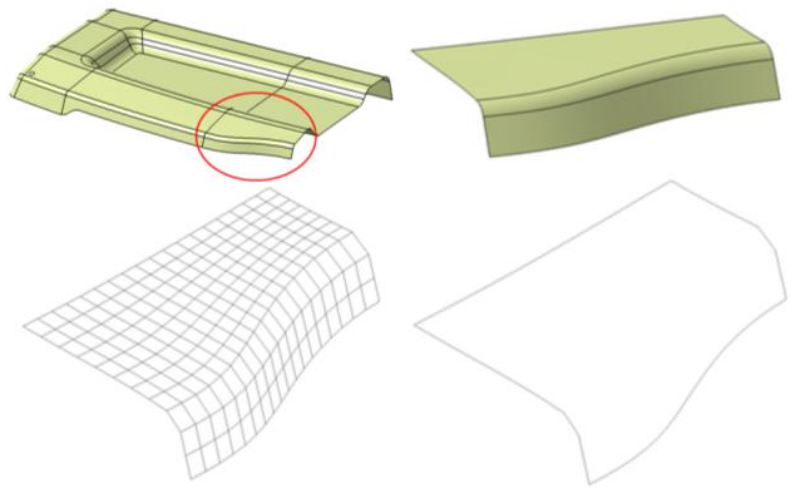

- $\quad$ Figure 2 : after the application of rules

\section{MASS OPTIMIZATION EXAMPLE}

If we consider mechanical engineering, we can consider three major kind of design optimization : Parametric, Geometric, Topological.

\section{A. Parametric design optimization (Size)}

Shapes are parameterized by variables like sections, thicknesses, diameters, length etc. This optimization does not give the possibility to explore new forms but it is only used to size the existing forms.

The optimization problem is for example to minimize the structural mass of a beam allowing a limited vertical deflection of the loading end. The displacement is limited to $2.0 \mathrm{~mm}$. All thickness variables have a lower bound of $1 \mathrm{~mm}$ and an upper bound of $3 \mathrm{~mm}$.

\section{B. Geometric design optimization}

Geometric design optimization is used to vary the boundaries of an original form without changing its topology. Changing the boundaries of the geometry requires updating the mesh. This remeshing is produced in the optimization process. The optimization problem is for example to maximize the stiffness of a plate by changing the shape of a curve. The thickness of the plate remains fixed. We want also to have the $\mathrm{X}$ displacement of the application's point of the force below $0,1 \mathrm{~mm}$ and the $\mathrm{Y}$ displacement below $0,08 \mathrm{~mm}$.

\section{Topology optimization}

The variable is the topology of the part. In this kind of optimization it may have appearance or disappearance of holes, reinforcement or changes in connections between elements. Topology optimization is an appropriate method for the early design phase of a new project because it allows exploring new design concepts for structures.

\section{Industrial example : a hook topology optimization}

This example is easy to understand (we have illustrated more complex examples in [3])but it is very interesting because it comes from a heavy hook ( $8 \mathrm{kgs})$ to an optimized hook $(5 \mathrm{kgs})$ without influencing too much the manufacturing process (foundry).

The examples below show the hook in its "real life", the optimization process (topology) and the physical test which concludes to the respect of the conditions. 

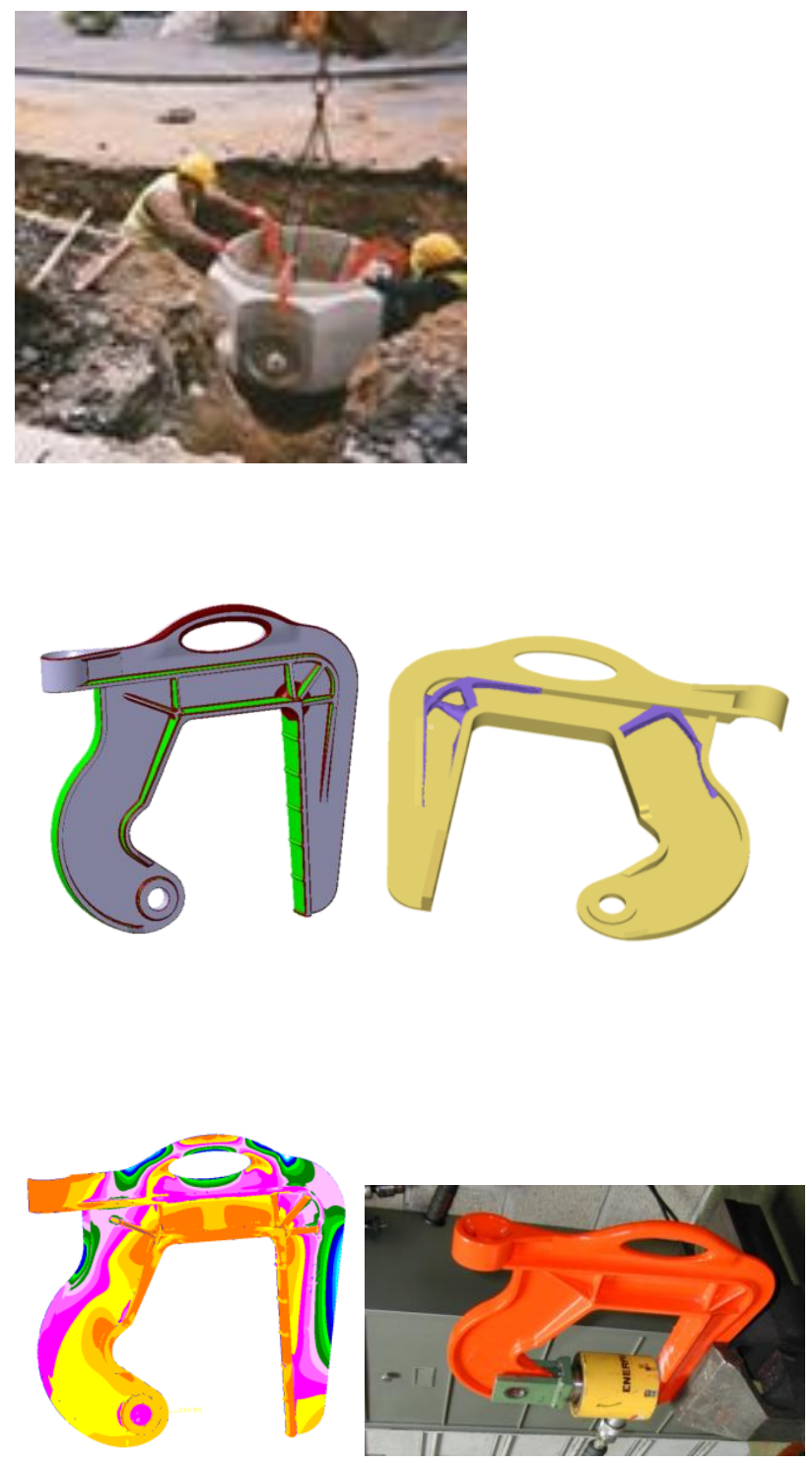

- Figure 3 : the example of the hook

\section{FIRTS PHASES OF FORGING OPTTIMIZATION}

In this part, we present a knowledge-based optimization dedicated to hot forging [14].
Hot forging is a multiple stages plastic deformation of metal starting from an initial part - named billet.

The main difficulty of this process is to get each intermediate metal part - named "preform" - that is actually defined by blacksmiths thanks to forging rules and know-how. Our application supplies automatically the entire process: it reduces the $3 \mathrm{D}$ desired forged part into some appropriate slices thanks to morphological and geometrical criteria. For each slice, it extracts trade features and deforms the slice according to forging rules. Finally, it creates the preform by assembling the deformed slices. The application may iterate on this preform if necessary.

This study is mainly based on trade knowledge rules whose some examples (rules 1 to 3 ) are given below.

rule 1 :

while (slope $<12 \%$ ) :

Improve the slope ;

Maintain the area ;

End while

rule 2 :

while (rayon $<10 \mathrm{~mm}$ )

Improve radius ;

Maintain the area;

end while

rule 3 :

while (Modification)

if (preform hight $<$ raw hight) then improve hight if (preform width $>$ raw width) then reduce width Maintain the area ;

end while

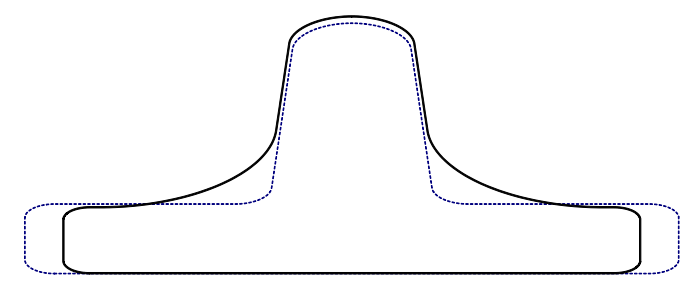

Application of rule 2

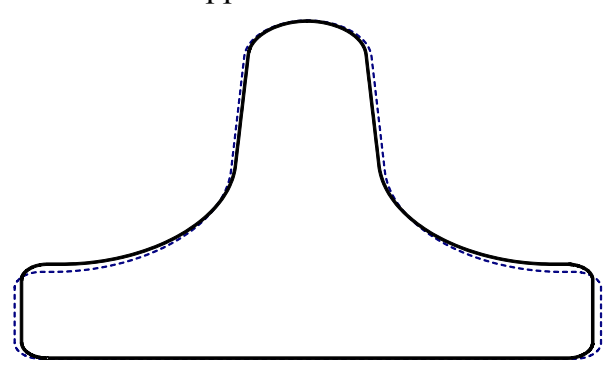

Application of rule 3 

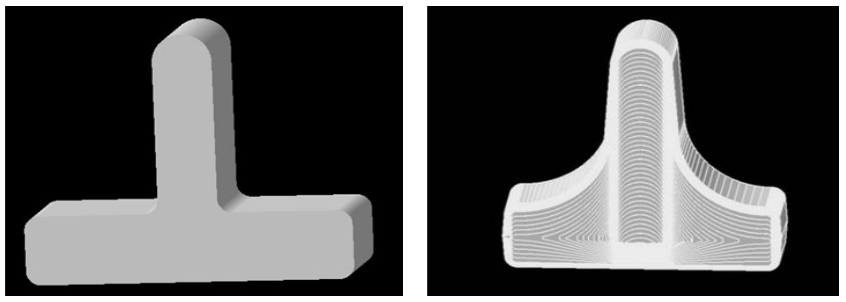

Forging raw (left) - optimized preform (right)

Figure 4 : preprocessing forging

It is a fact that giving the operator the opportunity to be helped by a dedicated software, ensuring that the trade rules are respected, will lead to optimized solutions.

We have extended this approach to take into account the possibility to "disrupt" certain parameters around the values given by an expert. It is based on the idea that parameters provided by experts or in guides give a "good" solution, but probably not the best solution.

So, it is easy by a parameter optimization (not on mechanical structures here) to propose new solutions.

In the foundry domain, we have then obtained new solutions for the filling system that are better (in term of lost material) of more than $40 \%$ compared to the solution given by the expert. It is done just by simulating small displacements of the parts.

\section{ADDITIVE MANUFACTURING OPTIMIZATION}

Rapid Prototyping (RP) and more generally Additive Manufacturing (AM) enable the manufacture of complex geometries which are very difficult to build with classical production. There are numerous technologies which are using different kind of material. For each of these, there at least two materials: the production material and the support one. Support material is, in most cases, cleaned and become a manufacturing residue. Improving the material volume and the global mass of the product is an essential aim surrounding the integration of simulation in additive manufacturing process. Moreover the layer by layer technology of additive manufacturing allows the design of innovative objects and the use of topological optimization in this context can create a very interesting combination.

We illustrate in this part how to take advantage of topological optimization for the preparation of model for RP and AM.

In topology optimisation design variable are element densities and are managed in our work by SIMP method. Specific techniques need to be introduced to penalize intermediate densities and to force final design to be represented by densities of 0 or 1 for each element. This particular adjustment is really important for the knowledge management. The penalization technique used is the power law representation of elasticity properties which can be expressed as shown on equation below where is the penalized stiffness matrix, $\mathrm{K}$ the real stiffness matrix, the density and $\mathrm{P}$ the penalization factor which has to be superior to 1 .

$$
\underline{K}(\rho)=\rho^{p} K
$$

This penalized factor will have a very relevant impact on our problem. Indeed, in the case or AM, we have to manage the minimum length which represent the thickness of the different walls. Belong to the machine process, this value is determined by experiment data. A simple example is shown on figure 5: on a simple C-CLIP optimisation, the penalization factor management allows a bad or a good management of knowledge [14].
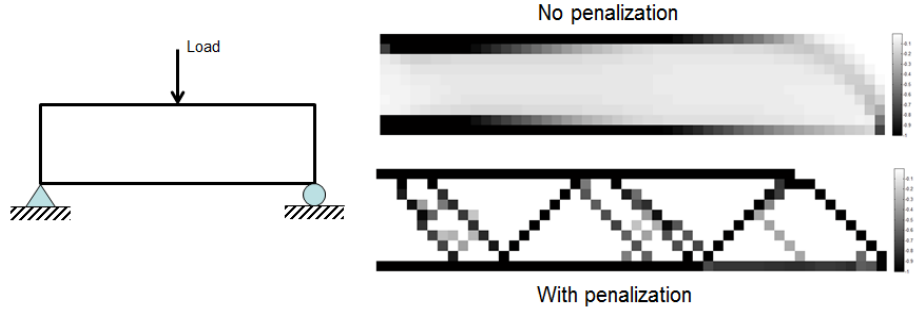

Figure 5 : penalization influence [14]

Our approach involves the study of three very important factors for the topological optimisation:

- The minimum thickness printable and cleanable without part deterioration. We seek to maximize the minimum thickness of the wire cloth (final material) without loss of geometric and morphological qualities of the part

- The minimum diameter printable and cleanable without mechanical cleaning: the objective is to size the best channels dimensions for cleaning the internal structure of the piece (allow the powder evacuation)

- The maximum height, in fact the ratio between the projected length and height of the part which may cause a falling down of the matter

We developed DOE (Design Of Experiment) for different tests: test of the laser temperature impact, test thickness and height allowed (with cleaning process), test of the manufacturing orientation, test of the plate placement ...

To validate our methodology and prepare the software integration, we first verified our assertion with commercial software. We developed in Rhinoceros3D an interface which helps the designer to prepare the CAD model and launch in 
background Optistruct solver. The program is developed in python. We study a prosthetic implant used in a hip replacement surgical procedure studied for one of our client (a simplify one with regard to the confidentiality). There are a large number of hip implant devices on the market. Many different shapes exist but each styles falls into one of four basic material categories metal on plastic, metal on metal, ceramic on plastic and ceramic on ceramic. Due to the history of our region (large of foundry and forge industrial impact), we are interested in the metal on metal material and more particularly on titanium. This kind of prosthesis is built with forge process in titanium material.

Previous work enabled us to integrate knowledge to CAD model. The modelling is based on a specific methodology briefly presented before that covers the knowledge capitalisation and modelling using scripts. The program which support the methodology is developed in python and is dedicated to the preparation of optimisation model [14].
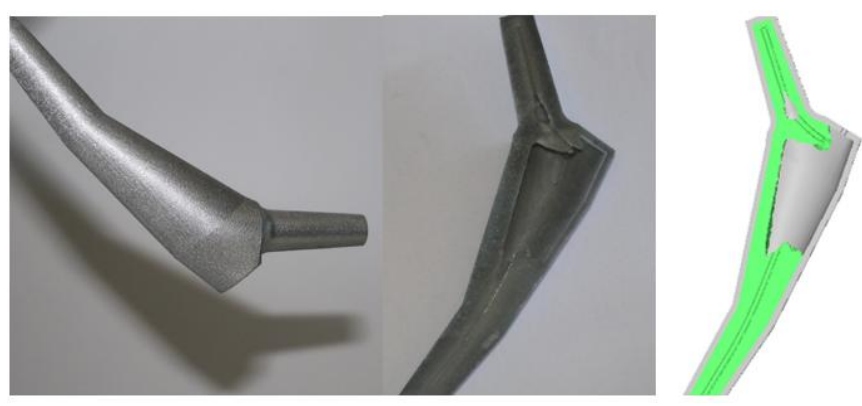

Figure 6 : optimization example for additive manufacturing

\section{CONCLUSION}

The goal of this paper was to illustrate the fact that local optimization cannot be considered without taking into account the entire process. Based on a "synthetic" approach and industrial examples, we have shown that many factors, from different points of views, are important. By carefully looking at them following a "synthetic" methodology it is possible to hugely improve the product and the process by using mathematical models linked with the trade knowledge.

\section{ACKNOWLEDGMENT}

This work has been partly funded by : OSEO, Region Champagne-Ardennes, FEDER (Europe) and Conseil Général des Ardennes.

\section{REFERENCES}

[1] Madux, K. C., Jain, S.C. "CAE for the manufacturing engineer: the role of process simulation in concurrent engineering", Manufacturing Simulation and Processes, ASME, 20, 1-15, 1986

[2] Sharpe, J.E.E. "Computer Tools for Integrated Conceptual Design ", Design Studies, 16(4), 471-488, 1995

[3] Yvon Gardan, Anthony Lerouge, Nicolas Gardan, and Elvis Kwassi. "A methodology to optimize design : fundamentals and case study". the 13th International conference on Mathematical and computational methods

[4] Roy, R., Hinduja, S., Teti, R., "Recent advances in engineering design optimization: Challenges and future trends", CIRP Annals, Manufacturing technology, 2008

[5] Danesi, F. Gardan, N. Gardan Y. Reimeringer, M. "P4 LM: A methodology for product lifecycle management", Computers in Industry 59 (2008) 304-317

[6] Arora, J., Introduction to Optimum Design, McGraw-Hill, 1989.

[7] Bendsoe, M. P., Sigmund, O., Topology Optimization, Theory, Methods and Applications, Springer, 2003

[8] Haftka, R. T., Guerdal, Z., Elements of Structural Optimization, Kluwer Academic Publishers, 1996.

[9] Marcelin, J. L., "Optimisation des structures et d'éléments mécaniques", Cépaduès, Toulouse, 2006

[10] N. Gardan, M. Reimeringer, F. Danesi, Y. Gardan "Graphonumerical parameters: collaborative parameters based on scenarios and ontologies" Proceedings of the PLM07, 11-13 Juillet 2007, KilometroRosso, Italie

[11] Nicolas Gardan, Yvon Gardan "An application of knowledge based modelling using scripts ", Expert Systems With Applications, Pergamon press, Elsevier Science Ltd., Volume 25, Issue 4, November 2003, Pages 555-568

[12] Michael Reimeringer, Nicolas Gardan, Yvon Gardan, Hassan Ugur "CADFORSIM : Rules to improve the mesh quality" — Proceedings of the TMCE 2008, April 21-25, 2008, Izmir, Turkey

[13] E. Brosse, Y. Gardan, E. Perrin - "Forging-dedicated CAD: how getting forging preform by using trade knowledge and feature reorganization" 4th International Conference Computer Graphics, Imaging and Visualization 14, 15 - 17 August 2007• Bangkok • Thailand, 2007

[14] Nicolas Gardan, Alexandre Schneider, "Topological optimization of internal patterns and support in additive manufacturing" - unpublished

\section{Creative Commons Attribution License 4.0 (Attribution 4.0 International, CC BY 4.0)}

This article is published under the terms of the Creative Commons Attribution License 4.0 https://creativecommons.org/licenses/by/4.0/deed.en_US 